
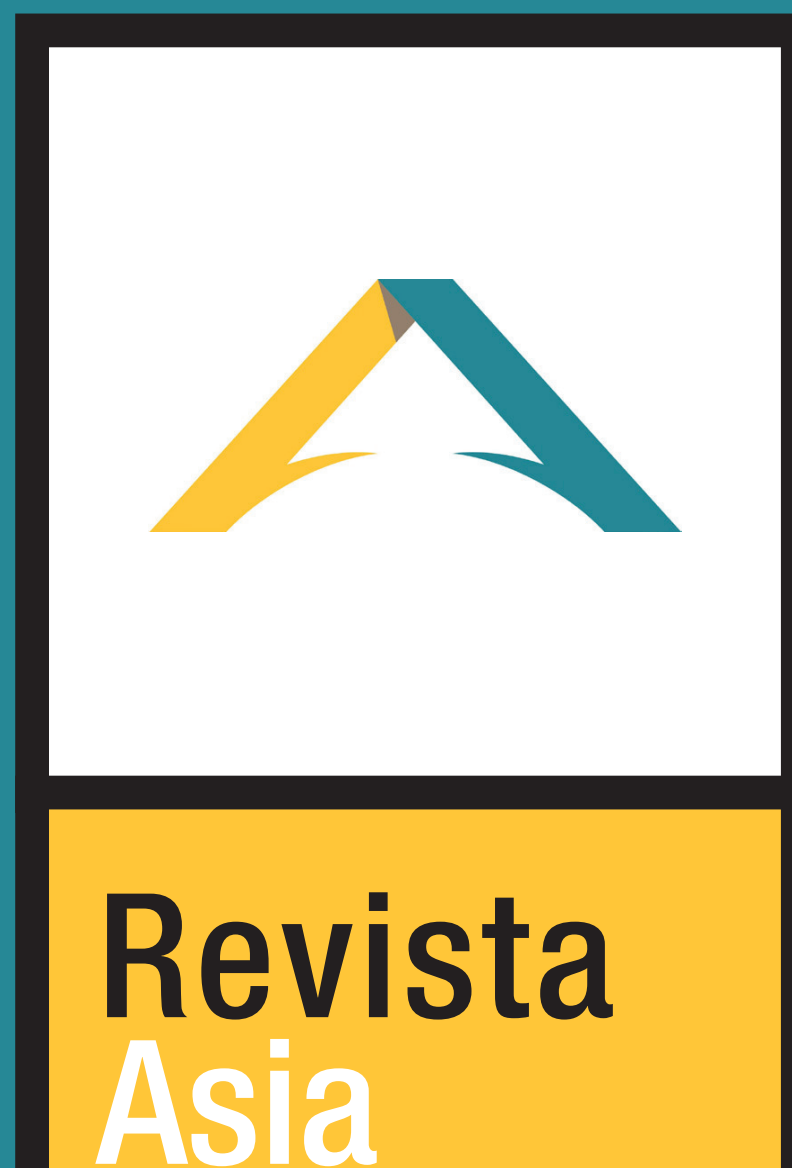

América Latina

Año 1. Volumen 1. Número 1. JUNIO 2016. Argentina ISSN 2524-9347

Grupo de Estudios de Asia y América Latina Instituto de Estudios sobre América Latina y el Caribe Universidad de Buenos Aires

\section{PRESENTACIÓN}

¿POR QUÉ UNA REVISTA SOBRE ASIA Y

AMERICA LATINA?

Fernando Pedrosa 5

\section{DOSSIER}

EL AMOR EN DOS LEYENDAS DEL SUDESTE ASIÁTICO

Mireya Sosa Abella 13

LA POLÍTICA EXTERIOR DE ARGENTINA HACIA TAILANDIA: CONDICIONAMIENTOS POLÍTICOS Y ECONÓMICOS DENTRO DE UNA ESTRATEGIA DE DIVERSIFICACIÓN
M. Florencia Rubiolo
29

INTERNACIONALIZACIÓN Y DIPLOMACIA GASTRONÓMICA EN TAILANDIA

Ezequiel Ramoneda 49

BREVE ANÁLISIS COMPARATIVO DE LA CAPOEIRA BRASILERA Y LA MÚSICA SAMARA TAILANDESA Y LAS PRÁCTICAS RITUALES COMPARTIDAS

Duncan Williams

67

\section{VARIA}

LOS DESAFÍOS POLÍTICOS EN CHINA HOY

Cristina Reigadas

87

\section{DIÁLOGOS}

¿QUÉ HACEMOS CON LOS VIEJOS? UNA SIMPLE PREGUNTA NO TIENE POR QUÉ TENER SIMPLE RESPUESTA: CHINA

Diego Bernardini 105 


\section{Dirección}

Dr. Fernando Pedrosa

(Grupo de Estudios de Asia y América Latina, Instituto de Estudios sobre América Latina y el Caribe, Universidad de Buenos Aires, Argentina)

\section{Secretaría de Redacción}

\section{Mg. Cecilia Noce}

(Grupo de Estudios de Asia y América Latina, Instituto de Estudios sobre América Latina y el Caribe, Universidad de Buenos Aires, Argentina)

\section{Dr. Ariel Sribman}

(Universidad de Girona, España)

\section{Consejo Editorial}

\section{Dra. Mercedes Botto}

(FLACSO-Argentina)

Dra. María José Bruña

(Universidad de Salamanca, España)

Dra. Pasuree Luesakul

(Directora del Centro de Estudios Latinoamericanos, Universidad de Chulalongkorn, Tailandia).

\section{Lic. Patricia Piccolini}

(Directora Carrera de Edición, Facultad de Filosofía y Letras, Universidad de Buenos Aires, Argentina).

\section{Lic. Ezequiel Ramoneda}

(Centro de Estudios del Sudeste asiático. Instituto de Relaciones Internacionales Universidad Nacional de La Plata, Argentina).

Dra. Cristina Reigadas

(Instituto Gino Germani, Facultad de Ciencias Sociales, Universidad de Buenos Aires, Argentina)

Dra. Florencia Rubiolo

(Universidad Nacional de Córdoba, Argentina)

Dra. Mireya Sosa Abella

(Universidad de Malasia)

Dr. Jaime Moreno Tejada

(Universidad de Chulalongkorn, Tailandia)

Dr. Ignacio Tredici

(ex Jefe del Equipo Jurídico de la Oficina del Co-Juez de Instrucción Internacional del Tribunal Khmer Rojo, United Nations Mission of Assistance to the Khmer Rouge Trials, Extraordinary Chambers in the Courts of Cambodia)

Dr. Wasana Wongsurabat

(Universidad de Chulalongkorn, Tailandia)

\section{Eudeba}

Universidad de Buenos Aires

$1^{\circ}$ edición: junio 2016

(C) 2016

Editorial Universitaria de Buenos Aires

Sociedad de Economía Mixta

Av. Rivadavia 1571/73 (1033) Ciudad de Buenos Aires

Tel: 4383-8025 / Fax: 4383-2202

www.eudeba.com.ar

DG: Alessandrini \& Salzman para Eudeba.

Impreso en Argentina

Hecho el depósito que establece la ley 11.723

No se permite la reproducción total o parcial de este libro, ni su almacenamiento en un sistema informático, ni su transmisión en cualquier forma o por cualquier medio, electrónico, mecánico, fotocopia u otros métodos, sin el permiso previo del editor. 


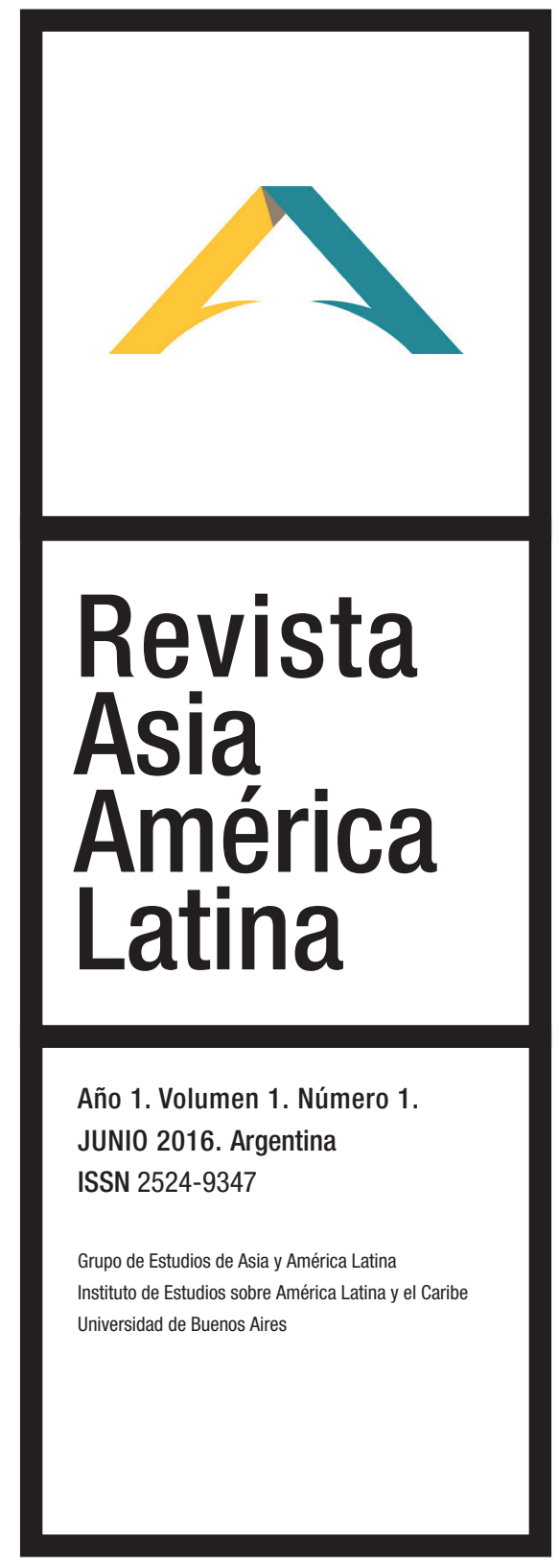

\section{PRESENTACIÓN}

¿POR QUÉ UNA REVISTA SOBRE ASIA Y AMERICA

LATINA?

Fernando Pedrosa 5

\section{DOSSIER}

EL AMOR EN DOS LEYENDAS DEL SUDESTE

ASIÁTICO

Mireya Sosa Abella 13

LA POLÍTICA EXTERIOR DE ARGENTINA HACIA TAILANDIA: CONDICIONAMIENTOS POLÍTICOS Y ECONÓMICOS DENTRO DE UNA ESTRATEGIA DE DIVERSIFICACIÓN

M. Florencia Rubiolo 29

INTERNACIONALIZACIÓN Y DIPLOMACIA GASTRONÓMICA EN TAILANDIA

Ezequiel Ramoneda 49

BREVE ANÁLISIS COMPARATIVO DE LA CAPOEIRA BRASILERA Y LA MÚSICA SAMARA TAILANDESA Y LAS PRÁCTICAS RITUALES COMPARTIDAS

Duncan Williams 67

\section{VARIA}

LOS DESAFÍOS POLÍTICOS EN CHINA HOY

Cristina Reigadas 87

\section{DIÁLOGOS}

¿QUÉ HACEMOS CON LOS VIEJOS? UNA SIMPLE PREGUNTA NO TIENE POR QUÉ TENER SIMPLE RESPUESTA: CHINA

Diego Bernardini 105

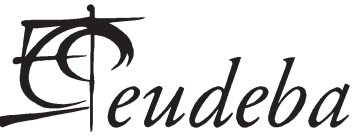





\title{
INTERNACIONALIZACIÓN Y DIPLOMACIA GASTRONÓMICA EN TAILANDIA
}

\author{
INTERNATIONALIZATION AND GASTRO- \\ DIPLOMACY IN THAILAND
}

\section{Ezequiel Ramoneda}

Centro de Estudios del Sudeste Asiático del Departamento de Asia y el Pacífico del Instituto de Relaciones Internacionales de la Universidad Nacional de la Plata.

ESEA, DAYP-IRI-UNLP

er131079@gmail.com

RESUMEN: El gobierno de Tailandia fue pionero en aprovechar el recurso no convencional de la popularidad de la comida tailandesa en el mundo e instrumentalizarlo estratégicamente dentro de las iniciativas diplomáticas llevadas adelante para relacionarse internacionalmente con otros estados. El presente trabajo busca hacer hincapié en uno de los aspectos culturales de la sociedad tailandesa: la gastronomía. De esta manera, se estudiará la internacionalización de la comida tailandesa como parte de una diplomacia gastronómica llevada adelante para aumentar el posicionamiento y conocimiento del país asiático. Para ello, el trabajo tratará sobre el proceso histórico de la internacionalización de la comida tailandesa, desde la época del reino de Ayutthaya (siglo XIV) hasta inicios del siglo XXI, con la implementación de una política de diplomacia cultural sostenida en su gastronomía.

Palabras clave: Tailandia, diplomacia gastronómica, internacionalización, comida.

ABSTRACT: The Thai Government was first to take advantage of the unconventional use of Thai food's worldwide popularity and strategically use it within the diplomatic initiatives developed to internationally relate with other States. This paper intends to focus on one of the cultural aspects of the Thai society: gastronomy. Thereby, it will study the internationalization of Thai food as part of a diplomatic gastronomy utilized to boost positioning and knowledge of the Asiatic country. To this end, this paper will focus on the historical process of internationalization of Thai food, since the time of Ayutthaya's Kingdom (XIV Century) until the beginning of XXI Century, with the use of a cultural diplomacy supported by its gastronomy

Keywords: Thailand, gastro-diplomacy, internationalization, food. 


\section{Introducción}

Argentina y Tailandia se encuentran en las antípodas del mundo, el primero en Sudamérica, el último en el Sudeste Asiático. Los contactos diplomáticos entre ambos países se iniciaron hace poco más de sesenta años a mediados de la década de 1950. Las relaciones comerciales, sin embargo, son mucho más recientes, desarrollándose en la década de 1990. A pesar de haber madurado las vinculaciones políticas y profundizado las relaciones comerciales, poco se sabe en Argentina en particular y en América Latina en general, de las particulares características sociales y culturales de Tailandia.

El presente trabajo busca hacer hincapié en uno de los aspectos culturales de la sociedad tailandesa: la gastronomía. De esta manera, se estudiará la internacionalización de la comida tailandesa como parte de una diplomacia gastronómica llevada adelante para aumentar el posicionamiento y conocimiento del país asiático. Para ello este trabajo tratará sobre el proceso histórico de la internacionalización de la comida tailandesa, desde la época del reino de Ayutthaya (siglo XIV) hasta inicios del siglo XXI, con la implementación de una política de diplomacia cultural sostenida en la gastronomía tailandesa ${ }^{1}$.

\section{La Comida Tailandesa "Conquista" Occidente: Internacionalización de la Comida Tailandesa}

Para entender parte del éxito de la diplomacia gastronómica del gobierno tailandés, hay que examinar el proceso de internacionalización de la comida tailandesa desde una perspectiva histórica. Es que a lo largo de los siglos, las tradiciones culinarias han sido parte de la vinculación del pueblo tailandés con el mundo.

Históricamente, la comida tailandesa ha sido producto de procesos dinámicos e interacciones culturales complejas. Una combinación de alimentos e ingredientes autóctonos y foráneos, de tradiciones gastronómicas indígenas con influencias culinarias -más que centenarias- proveniente de India y China, e incluso de Occidente $^{2}$. Esto dio como resultado que la gastronomía

1. La diplomacia gastronómica se distingue de la "diplomacia culinaria", porque esta última se caracteriza por la utilización protocolar de la comida en el desarrollo de actividades oficiales entre representantes diplomáticos de gobiernos. Se diferencia también de la "diplomacia alimentaria", a partir de la cual se brinda ayuda a otros estados en situaciones de crisis o catástrofes a través de la donación de alimentos (Rockower , 2012).

2. "Thailand Kitchen of the World", Foreign Office of the Government Public Relations Department of the Office of the Prime Minister of the Kingdom of Thailand, 2009.

Extraído de http://thailand.prd.go.th/ enero de 2016. 
tailandesa sea de una riqueza y complejidad caracterizada por el balance armonioso de los sabores dulce, salado, picante, amargo, y acido, que a su vez viene a representar la combinación de los elementos de naturaleza, tierra, agua, madera, fuego y metal (Sunanta, 2005).

El proceso de conocimiento y negociación entre los gobernantes tailandeses con los representantes diplomáticos de otros reinos, como también con comerciantes extranjeros, acontecía en un contexto gastronómico: interactuaban mientras compartían la comida. Los europeos no fueron la excepción ya que Tailandia tiene una larga historia de vinculaciones con los países occidentales.

Durante el reino de Ayutthaya (1351-1767) tuvieron lugar los primeros contactos de con los europeos, pero fue entrado el siglo XVI que llegaron formalmente las primeras potencias europeas. En primer lugar Portugal, seguida luego por Holanda. Poco tiempo después, debido al establecimiento de relaciones políticas y al crecimiento de las relaciones comerciales, los europeos establecieron barrios propios en las afueras de la ciudad capital.

A su vez, se debe señalar que este interés no fue unilateral por parte de los europeos, sino que también los tailandeses comenzaron a viajar hacia Europa. Durante el gobierno del rey tailandés Narai (1633-1688), se organizaron misiones diplomáticas para visitar Europa. Entre los años 1686 y 1687 esas misiones visitaron Inglaterra y Francia, llegando a reunirse con el rey Luis XIV. Luego, entre 1688 y1689, además de volver a Francia y reunirse con el rey Luis XIV, visitaron el Vaticano, donde se entrevistaron con el papa Inocencio $\mathrm{XI}^{3}$.

Para el siglo XIX, ya con el inicio de la expansión imperialista europea por Asia Oriental, las vinculaciones de las potencias europeas con el reino de Tailandia se profundizaron. Durante el reinado de Rama III se firmó un tratado con Inglaterra en 1826 y con los Estados Unidos en 1833. Durante el reinado de Rama IV, se firmaron nuevos tratados con Inglaterra en 1855 y con los Estados Unidos en 1856, a los cuales se sumaron posteriormente otras potencias occidentales. Rama $\mathrm{V}$ debió llevar adelante diversas negociaciones con las potencias occidentales, situación que lo llevó a realizar dos giras oficiales por países europeos, en 1897 y 1907, lo cual lo convirtió en el primer monarca tailandés en hacerlo.

Más allá del posible interés por lo exótico generado en algunos de los intercambios, para ese entonces la comida tailandesa no pudo superar las fronteras del país. Conspiraban contra ello diversos factores, como ser la

3. "The Beginning of Relations with European Nations and Japan", Bangkok, Ministry of Foreign Affairs of the Kingdom of Thailand. Extraído de http://www.mfa.go.th/ diciembre de 2015. 
naturaleza recluida y puntual de la interacción diplomática, cierta inadaptabilidad de la comida tailandesa, además de la ausencia de ingredientes originales en los países europeos.

Fue debido a la capacidad de los gobernantes de la dinastía Chakri, que el reino de Tailandia logró mantener su independencia y también avanzar en la modernización del país. Esta dinastía, especialmente personificada en los ya mencionados Rama IV y Rama V, logró que Tailandia haya sido el único pueblo del Sudeste Asiático que, aunque experimentó la influencia occidental, no fue colonizado por las potencias europeas (Panikkar, 1966).

De la misma manera, tampoco la comida tailandesa fue "colonizada" por Occidente, escapando así a la imposición de las preferencias y gustos europeos por sobre los de los locales. Sin embargo, esto no significó un rechazo total a esa influencia, por el contrario, implicó un proceso de elección y selección de determinados elementos de las gastronomías europeas de acuerdo a las propias preferencias de los tailandeses (Van Esterik, 2008).

En el proceso de modernización, los gobernantes sentaron también las bases para el desarrollo del turismo doméstico -llevado adelante por la aristocracia- y para el internacional. Desde fines del siglo XIX, Bangkok empezó a servir como puerto de escala para cruceros y otras grandes embarcaciones destinadas al transporte de mercancías y comunicaciones (Douglas y Douglas, 2011). La mayoría de los visitantes extranjeros eran ingleses y franceses que pasaban por Tailandia en viaje a las posesiones coloniales en la región, a saber Birmania y Malasia bajo el imperio Inglés, y Laos, Camboya y Vietnam bajo el imperio Francés (Kontogeorgopoulos, 1998).

Este aumento de los visitantes occidentales siguió ocurriendo en forma creciente hasta la segunda mitad del siglo XX. Con la violencia y devastación de la Segunda Guerra Mundial, la cantidad de visitantes provenientes de Europa descendieron. A pesar de haber participado en este enfrentamiento global como aliado de Japón contra Estados Unidos e Inglaterra, con el fin de la contienda en agosto de 1945, Tailandia se convirtió rápidamente en aliado estratégico de los Estados Unidos. Esto ocurrió en el marco de la emergencia de la Guerra Fría y la oposición tailandesa frente a la expansión del comunismo.

Durante la llamada guerra de Indochina (1946-1954), Tailandia apoyó las iniciativas militares de los Estados Unidos contra las fuerzas comunistas en el territorio vietnamita (Bianco, 1995). Esto trajo como consecuencia un aumento en la cantidad de visitantes estadounidenses que por ello, comenzaron a superar a los europeos. Incluso los norteamericanos llegaron a ser la mayoría entre los visitantes extranjeros de Tailandia.

En este contexto, tuvieron lugar una serie de procesos complementarios que permitirían el desarrollo masivo de la industria del turismo internacional en Tailandia (Van Esterik, 2008). El establecimiento del turismo como 
industria comenzó durante el gobierno del primer ministro Sarit Thanarat (1957-1963), quien implementó una serie de políticas para abrir y desarrollar la economía y fortalecer así significativamente la imagen internacional de Tailandia

Entre otras medidas se pueden mencionar la mejora de la infraestructura, la expansión del suministro de agua y electricidad, la actualización del transporte y las comunicaciones, la agilización de los servicios comerciales y bancarios, y el establecimiento de un marco institucional y regulatorio.

Lasmedidasincentivaron el crecimientoeintensificaron eldesarrollodel sector de servicios, dentro del cual se incluye el turismo (Kontogeorgopoulos, 1998). En 1960, se estableció la Organización de Turismo, luego renombrada en 1963 como la Organización de Turismo de Tailandia (TOT), la agencia gubernamental responsable de supervisar la promoción y la comercialización del país como destino turístico internacional ${ }^{4}$.

Otro aspecto a considerar es que, en la década de 1960, los tailandeses empezaron a emigrar a países occidentales en busca de trabajo o para realizar estudios, especialmente hacia Estados Unidos, junto con otros países occidentales como Canadá, Inglaterra y Australia. Estos migrantes llevaron consigo su cultura y sus creencias, y también su comida. De esta manera los países occidentales no solo fueron el origen principal de los visitantes a Tailandia, sino también el destino preferente de las migraciones tailandesas al mundo.

Paradojicamente, la guerra de Vietnam, continuación de la antes mencionada, impulsó la industria del turismo internacional en Tailandia. Estados Unidos no sólo inyectó grandes cantidades de recursos en la economía tailandesa sino que también estableció bases militares en el territorio. Estas bases dinamizaron la economía de las zonas circundantes al estimular el establecimiento y crecimiento de bares, restaurantes, clubes y otros negocios de servicios cuyas actividades estaban destinadas a satisfacer las necesidades de las tropas estadounidenses.

Según Meyer (1988), entre 1966 y 1974 estuvieron estacionados aproximadamente 321.800 soldados estadounidenses en las bases militares instaladas en el territorio tailandés, además de otros 310.400 que realizaron visitas a este país de recuperación y recreación del combate.

La prolongada presencia de las tropas estadounidenses durante las décadas de 1960 y de 1970 generó como resultado un conjunto de cambios, entre los cuales se destaca un proceso de occidentalización del sector de servicios para adecuarse a las necesidades de las tropas estadounidenses.

4. Incluso en 1965 se abrió la primera oficina en el extranjero con sede en la ciudad de Nueva York. 
Asimismo, se produjo un proceso de influencia de la cultura tailandesa sobre las tropas estadounidenses quienes mantuvieron algunas costumbres, incluida la comida, una vez regresados a Estados Unidos, en muchos casos de la mano de sus nuevas esposas.

Otro efecto de la guerra de Vietnam fue la difusión creciente de la imagen internacional de Tailandia, lo que también condujo a un incremento de los visitantes extranjeros. Aunque para estos años, los hoteles de altos estándares internacionales, como también los restaurantes de comida tailandesa en la misma Bangkok, no eran muy numerosos (Cohen 20o8; Van Esterik, 2008; Van Esterik, 2011), la experiencia turística produjo una mayor interacción cultural.

Esto resultó, entre otras cosas, en un desarrollo favorable a la incorporación de la comida tailandesa, en su versión palaciega, en el gusto de los visitantes occidentales. A la vez se profundizó un proceso de expansión más allá de las fronteras del país, comenzando a convertirse también en parte del repertorio gastronómico del mundo occidental (Kontogeorgopoulos, 1998; Sunanta, 2005).

Fuera de Tailandia el fenómeno también tendió a consolidarse. Algunos de los tailandeses en el extranjero, particularmente en América del Norte y los países de Europa Occidental, fueron quienes establecieron los primeros restaurantes de comida autóctona. En en la década de 1960, estos negocios estaban dedicados inicialmente para la comunidad inmigrante tailandesa, pero al poco tiempo, comenzaron a atraer a los consumidores occidentales.

Terminados los conflictos en la región asiática, y potenciada la estabilidad regional surgida por la conformación en agosto de 1967 de la Asociación de Naciones del Sudeste Asiático (conocida por sus siglas en ingles, ASEAN e integrada originalmente por Malasia, la República de Singapur, la República de Indonesia, y la República de Filipinas, además del Reino de Tailandia) y la firma en noviembre de 1971 de la declaración sobre la zona de paz, libertad y neutralidad por sus integrantes, los aportes económicos fruto de la presencia de las tropas estadounidenses en Tailandia descendieron.

Para paliar la situación, el gobierno tailandés mejoró la cantidad y calidad de los servicios turísticos, aprovechando la amplia diversidad de atracciones naturales, culturales, arqueológicas y religiosas, y los entretenimientos urbanos que dispone el país. Además de la característica hospitalidad del pueblo tailandés y el estilo de vida occidentalizado mantenido en los centros donde residieron las tropas estadounidenses. Como resultado, grandes cantidades de turistas extranjeros de todo el mundo llegaron al país hacia finales de la década de 1970 y principios de la década de 1980.

Reconociendo la importancia del turismo en la economía, el gobierno publicó en 1977 el primer Plan Quinquenal de Desarrollo del Turismo (19771981), que sirvió de guía para la elaboración e implementación de medidas en 
pos de fomentar el desarrollo del sector (Higham, 2000). En 1979, reformuló la TOT y estableció la Autoridad de Turismo de Tailandia (TAT), que se convirtió en la agencia gubernamental encargada de implementar las estrategias de promoción y comercialización turística para hacer de Tailandia uno de los destinos turísticos más reconocidos internacionalmente en el mundo.

En 1980, se lanzó la primera campaña turística bajo el slogan "Visit Thailand" ("Visita Tailandia"), relanzada en 1987. Desde entonces, los arribos de visitantes aumentaron explosivamente lo que condujo a la masificación de la industria del turismo en Tailandia. A los visitantes provenientes de Estados Unidos y Europa occidental, especialmente Inglaterra y Francia, se sumaron para ese entonces visitantes de otros países asiáticos (especialmente Singapur, Malasia, Japón, Corea del Sur, Hong Kong, Taiwán, y China) los cuales llegaron a ser la nueva mayoría de turistas (Cohen, 2008; Higham, 2000).

A diferencia de los primeros momentos en que contó con apoyo real o estatal, este éxito de la industria del turismo en Tailandia se basó sobre todo en la iniciativa del sector privado. Otro factor que ayudó en el crecimiento acelerado del sector fue un contexto de fuerte expansión general del turismo internacional que se observó a escala regional, el cual impulsó el desarrollo del turismo en el Sudeste Asiático en general y en Tailandia en particular.

Las autoridades buscaron consolidar al país como el centro de servicios y puerta de entrada del turismo internacional para el Sudeste Asiático continental (Myanmar, Laos, Camboya, Vietnam, y, especialmente, China Meridional). Esto también se potenció con la firma de los acuerdos de libre comercio firmados desde inicios de la década de 1990, empezando con el acuerdo de libre comercio de ASEAN de 1992, el cual asentó las bases para la movilidad de las personas entre los países de la región, junto con la circulación de bienes y servicios. (Higham, 2000; Kontogeorgopoulos, 1998).

Según datos del Tourism Authority of Thailand los arribos de visitantes extranjeros anuales crecieron aproximadamente de 81.00o turistas en 1960 a 7.192.00o turistas en $1996^{5}$ (Cuadro 1), mientras que los ingresos derivados crecieron de US\$ 8 millones en 1960 a US\$8.700 millones en 1996 (Kontogeorgopoulos, 1998).

El sector turístico internacional ha implicado cuantiosos beneficios para la economía de Tailandia, siendo uno de los que más aportan al producto bruto interno del país. Según datos de la Tourism Authority of Thailand, entrada la década de 1980 la industria del turismo se constituyó en la principal

5. "History", Bangkok, Tourism Authority of Thailand. Extraído de http://www.tatnews. org/ en diciembre de 2015. 
Asia

fuente de generación de ingresos de divisas del país, superando a todas las exportaciones (Cuadro 2).

Cuadro 1. Cantidad de Arribos Internacionales (1960-2006)

\begin{tabular}{ll}
\hline Year & Number of Arrivals \\
\hline 1960 & 81,340 \\
1965 & 225,025 \\
1970 & 628,671 \\
1975 & $1,180,075$ \\
1980 & $1,858,801$ \\
1985 & $2,438,270$ \\
1990 & $5,298,860$ \\
1995 & $6,951,566$ \\
2000 & $9,508,623$ \\
2005 & $11,516,936$ \\
2006 & $13,821,802$ \\
\hline
\end{tabular}

(Cohen, 2008, p.3)

Cuadro 2. Principales exportaciones e ingresos de Tailandia (1981-1989)

\begin{tabular}{|c|c|c|c|c|c|c|c|c|c|c|}
\hline \multirow[t]{2}{*}{ Rank } & \multicolumn{2}{|c|}{1981} & \multicolumn{2}{|c|}{1983} & \multicolumn{2}{|c|}{1985} & \multicolumn{2}{|c|}{1987} & \multicolumn{2}{|c|}{1989} \\
\hline & Export & Value & Export & Value & Export & Value & Export & Value & Export & Value \\
\hline 1 & Rice & 26367 & Tourism & 25050 & Tourism & 31768 & Tourism & 50024 & Tourism & 96383 \\
\hline 2 & Tourism & 21455 & Rice & 20157 & Textiles & 23578 & Textiles & 48555 & Textiles & 74036 \\
\hline 3 & Tapioca & 16446 & Tapioca & 15387 & Rice & 22524 & Rice & 22703 & Rice & 45462 \\
\hline 4 & Textiles & 12531 & Textiles & 14351 & Tapioca & 14969 & Tapioca & 20661 & Rubber & 26423 \\
\hline 5 & Rubber & 10840 & Rubber & 11787 & Rubber & 13567 & Rubber & 20539 & Tapioca & 23974 \\
\hline
\end{tabular}

(Lan Li Wei Zhang, 1997; Higham, 2000, p.131)

Al desarrollo de la industria del turismo, desde la década de 1960 hasta la actualidad, se debe agregar, como tambipen se mencionó con anterioridad, el proceso de la emigración a los países occidentales. De acuerdo al censo del 2010, la población originaria o descendiente de tailandeses más grande fuera del país se encuentra en los Estados Unidos, y se estima en 237 mil personas (Hoeffel, Rastogi, Kim y Shahid, 2012).

La misma se encuentra fundamentalmente concentrada en la ciudad de Los Ángeles, donde se encuentra el primer barrio tailandés del mundo, reconocido como tal en 1999. Otras comunidades importantes son las de Australia, que de acuerdo al censo del 2011 se estima en 45 mil personas ${ }^{6}$. Esta comunidad se encuentra concentrada, sobre todo, en la ciudad de Sydney,

6. "Community Information Summary: Thailand-born". Canberra, Department of Immigration and Citizenship of the Australian Government, 2014. Extraído de https:// www.dss.gov.au/ en diciembre de 2015. 
donde se encuentra el segundo barrio tailandés más importante del mundo (Beasley, Hirsch, y Rungmanee, 2014). En tercer lugar sigue Inglaterra con una población calculada en aproximadamente 40 mil personas 7.

Como efecto colateral de desarrollo de estos aspectos, el proceso histórico de internacionalización de la comida tailandesa en Occidente aumentó significativamente. La diáspora tailandesa generó el mercado básico, el cual fue potenciado por la clientela occidental. En las décadas de 1970 y de 1980 había unas pocas decenas de restaurantes de comida tailandesa fuera del país (Van Esterik, 2008). Para el 2002 existían aproximadamente 5.500 en el mundo ${ }^{8}$.

Según datos del Banco de Exportaciones-Importaciones de Tailandia, para el 2003 existían aproximadamente 6.875 restaurantes de comida tailandesa en el extranjero: 49\% de ellos estaban en los Estados Unidos y Canadá; $20 \%$ en Europa, $15 \%$ en Australia y Nueva Zelanda, el 14\% en Asia y el 2\% en otros países (Sunanta 2005).

\section{La Comida Tailandesa "acerca" a Occidente: diplomacia gastronómica del Gobierno Tailandés}

El turismo en Tailandia es un fenómeno vasto, multifacético y complejo. $\mathrm{Y}$ poco se ha estudiado las relaciones del mismo con contextos sociales $\mathrm{y}$ políticos más amplios. (Cohen 2008) Con este espíritu, las paginas siguientes buscan abordar la vinculación entre el desarrollo de la industria del turismo internacional en Tailandia y la internacionalización de su gastronomía (Sunanta, 2005).

Planteado esto, se pretende analizar las implicancias políticas y, especialmente diplomáticasde esta vinculación A principios de la década de 2000, tuvo lugar un aprovechamiento de la popularidad internacional de la comida tailandesa por parte del gobierno tailandés, resaltándolo y apuntalándolo por medio de la diplomacia para la promoción del turismo.

En este sentido el gobierno tailandés ha aprovechado la comida nacional mejor que la mayoría de los países del Sudeste Asiático (Van Esterik 2008). Así Tailandia se convirtió en un uno de los estados pioneros en el mundo en implementar una política de diplomacia cultural basada en la gastronomía, actualmente una de las más desarrolladas, integrales y exitosas.

7. "2011 Census: Quick Statistics for England and Wales on National Identity, Passports Held and Country of Birth". Office for National Statistics (2013), London, UK Statistics authority. Extraído de http://www.ons.gov.uk/ en diciembre de 2015.

8. "Food as Ambassador: Thailand's Gastro Diplomacy". The Economist, February, 2002. Extraído de http://www.economist.com/ en diciembre de 2015. 
Antes de analizar específicamente la diplomacia gastronómica del gobierno tailandés, es necesario plantear lo que se entiende por dicho concepto. Existe una íntima conexión entre la comida y la diplomacia en el proceso de interacción entre los gobernantes de distintos pueblos. La utilización de ingredientes específicos producto de un entorno natural concreto, las técnicas de preparación y cocción, los sabores, los aromas, y las textura, la manera de presentarlos y servirlos, y las formas de consumirlos, todas estas cuestiones relacionadas son parte del acervo cultural de un pueblo.

A su vez la comida es una instancia de sociabilidad, donde se transmite ese acervo cultural al compartir la experiencia de la comensalidad. Así, cuando gobernantes de distintos pueblos se sientan y comparten la comida, comparten entre ellos formas de sus respectivas culturas. (Juyan Zhang, 2015).

Según Anderson (1993), la concepción de la identidad nacional es fundamentalmente una construcción social simbólica, sin esencia determinada. Esta identidad se ha desarrollado durante la historia, producto del proceso creativo dinámico de imaginarla por parte de quienes detentan el poder para reunir y cohesionar a las personas en la pertenencia a una nación. Sin embargo, que sea una elaboración imaginaria no significa que sea irreal, sino que es intangible, y se realiza en un espacio mental donde se encuentran las creencias y tradiciones, pensamientos y emociones.

La historia es su principal fuente, donde se encuentran los elementos y las características concretas enraizadas en creencias y tradiciones en las que se sustentan los símbolos (Anderson, 1993). Según Hobsbawm (2002) los gobernantes de los Estados modernos reinterpretaron y remodelaron la historia por medio de la invención de las tradiciones.

Reimaginar la identidad nacional de un pueblo, seleccionando y resaltando determinados elementos y características específicas de la historia y la tradición, al mismo tiempo que eliminan y relegan otras. Con esta operación buscaban insertarse a ellos mismos como parte integrante de esta memoria y consciencia colectiva, creando una apariencia de continuidad.

El momento en que la invención de tradiciones tiene lugar es el momento en que surge la concepción de la identidad nacional del pueblo (Hobsbawn, 2002). En la época de los Estados-Nación modernos de fines del siglo XIX y siglo XX, elementos de la cultura de un pueblo se constituyeron en parte de su identidad nacional, dentro de los cuales estaba la comida, por lo que los estados pudieron estimular los sentimientos nacionales por medio de la gastronomía, lo que podría llamarse un nacionalismo gastronómico (Ferguson, 2004).

Tailandia no fue la excepción. Pero a la vez, socialmente, también existen diferencias, ya que podemos encontrar una cocina de clase alta o de elite, caracterizada por la complejidad de su elaboración y el refinamiento en la 
decoración de los platos preparados en el palacio, y una cocina popular, de características más sencillas (Sunanta 2005; Juyan Zhang, 2015).

Fue la cocina del palacio real durante el reinado de Rama II donde se perfeccionaron el arte de cocinar y comer, y sus creaciones culinarias establecieron el ejemplo a seguir. Luego, durante el reinado de Rama V, se compiló y publicó el primer libro de recetas tailandesas. ${ }^{9}$

$\mathrm{Al}$ seleccionar unos platos sobre otros dentro de esta diversidad y al generar la apariencia de homogeneidad y autenticidad a partir de esta selección, los gobernantes tailandeses aportaron cuidadosamente a través de la experiencia de los sabores, los aromas y las texturas de la comida a la constitución política de los rasgos auténticos, distintivos y exclusivos de la identidad nacional, la tailandinidad, por medio de una tradición inventada (Sunanta, 2005).

Ahora bien, los Estados-nación modernos también pudieron estimular los sentimientos nacionales por medio de la difusión de la gastronomía a nivel internacional. La popularidad internacional de la comida tailandesa, como parte del acervo nacional, se traduce en el reconocimiento de su cultura, lo que aumenta el orgullo nacional (Van Esterik, 2008). Según la primer ministro Yingluck Shinawatra, "El pueblo Thai está orgulloso de la rica tradición y popularidad global de la comida Tailandesa, la cual ha ayudado a promover la cultura Thai" ${ }^{10}$ Recién a principios del siglo XXI, la interacción entre comida, cultura, nación y diplomacia obtuvo reconocimiento propio dentro del ámbito de las relaciones internacionales.

Entre los recursos no convencionales que utilizan los gobiernos al establecer y desarrollar relaciones internacionales con otros estados, se encuentran la interacción de aspectos culturales o el intercambio de los elementos culturales intangibles. La comida, al ser parte del acervo cultural de la identidad nacional del país que puede ser desterritorializada fácilmente, es uno de estos.

Inspirado en el concepto elaborado por Nye (2009) de "poder blando" para explicar la capacidad de influenciar y atraer a otros por medios culturales. Rockower (2012) ha definido la "diplomacia gastronómica" como el acto de ganarse los corazones y las mentes de las personas a través del estómago. Esta instrumentalización estratégica de la comida nacional de un país, como herramienta en el ejercicio de la diplomacia cultural, permite promover la

9. Compilado por Plian Phasakorawong. (Foreign Office of the Government Public Relations Department, año).

10. "Welcoming Address by Her Excellency Ms. Yingluck Shinawatra, Prime Minister of the Kingdom of Thailand at the Launch of "Thailand : Kitchen to the World" Food Week, 27 May 2012, Shangri-La Hotel, Sydney", Bangkok, Ministry of Foreign Affairs of the Kingdom of Thailand. Extraído de http://www.mfa.go.th en diciembre de 2015. 
apreciación, el conocimiento y la comprensión que se tiene de la cultura de un país por parte de otros pueblos. Así se forman conexiones emocionales con la cultura por medio de la experiencia sensorial de la comida.

De esta manera, se fortalece el poder blando del Estado en cuestión, pudiendo persuadir e influenciar convenientemente a pueblos extranjeros, indirectamente reforzando actitudes positivas en aquellos. Consecuentemente, esta influencia positiva se extenderá a los estados que los representan, pudiendo mejorar así las relaciones entre los países en cuestión. Esto puede redundar positivamente ademas, en la construcción de una reputación internacional y global positiva del país más allá de las relaciones bilaterales entre estados (Rockower, 2012).

El gobierno del Primer Ministro Thaksin Shinawatra (2001-2006) introdujo una serie de medidas orientadas estratégicamente a sectores específicos del poder blando por considerarlos complementarios y de este modo fomentar una sinergia entre ellos (Intarakumnerd, 2011).

Una política turística más comprehensiva e integral fue implementada a partir de entonces. No solo se trataba de mejorar la posición de Tailandia en Asia, Thaksin buscó convertir al país en la "Capital del turismo de Asia", para incrementar significativamente el arribo de visitantes extranjeros.

A su vez, tratando de incrementar los ingresos derivados del turismo, Thaksin orientó a esta industria para priorizar el desarrollo del turismo de lujo, de modo de estimular el incremento de los gastos de los turistas extranjeros. Así, durante el gobierno de Thaksin, el turismo internacional de Tailandia vivió una etapa de gran crecimiento (Cohen, 2008).

Dentro de las iniciativas mencionadas, en el año 2002 el gobierno tailandés, con la participación de varios ministerios, lanzó el programa "Global Thai", el cual es reconocido como la primera iniciativa de diplomacia gastronómica integral conducida por un gobierno en el mundo (Rockower, 2012). El objetivo principal de esta innovadora iniciativa era incrementar internacionalmente la cantidad de restaurantes de comida tailandesa para fomentar y facilitar que los consumidores extranjeros se familiaricen y que ello luego estimulara un mayor interés por el turismo.

La versión de la comida tailandesa que la mayoría de los consumidores occidentales conoce, representa -socialmente- la comida en su versión palaciega. También es una versión de la comida de Bangkok y el centro del país, que es el punto de entrada y el atractivo principal a nivel del turístico internacional. De aquí que haya cierta homogeneización en los menús de los restaurantes de comida tailandesa en el exterior, orientado a lo que los consumidores occidentales no tailandeses conocen (Sunanta, 2005).

Los clientes extranjeros de un restaurante de comida tailandesa no sólo consumen la comida sintiendo sus sabores, aromas y texturas sino también se integran en establecimientos decorados con pinturas e imágenes alusivas, 
ambientados con aromas y música afines, y ser atendidos por personal vestido en trajes tradicionales que siven sus platos en vajilla típica. Se crea así una atmósfera y se experimenta vívidamente la auténtica cultura tailandesa en sus respectivos países. (Nirwandy y Awang 2013; Sunanta, 2005). De esta manera, los restaurantes se convierten en un "escaparate" donde promocionar el país.

Para potenciar esto, el gobierno buscó incentivar a los emprendedores para invertir en nuevos restaurantes ofreciendo préstamos flexibles con bajas tasas de interés. Este prestamo servía tanto para la financiación del establecimiento como para la contratación de chef tailandeses o con experiencia certificada. También para brindar entrenamiento en las escuelas de cocina más prestigiosas del país, como la Dusit Thani College, la Escuela de Cocina Internacional Suan Dusit de la Universidad de Rajabhat, el Instituto de Tecnología Rajamangala, y la Escuela de Cocina Wandee.

De este modo se buscaba que los participantes apropiadamente aprendan las sutilezas y conozcan los secretos de la comida tailandesa, fomentar la utilización de ingredientes originales importados de Tailandia.

También organizando demostraciones y degustaciones de comita tailandesa, con la participación de chef tailandeses invitados por las representaciones diplomáticas de los países en que se organizaban festivales, ferias o exposiciones internacionales. Como resultado de esta programa innovador, según Chapple-Sokol (2013), para el 2009 habría aproximadamente 13.000 restaurantes tailandeses fuera de Tailandia (Ichijo y Ranta, 2015).

En forma complementaria y producto del surgimiento descontrolado locales de comida tailandesa por el mundo, el gobierno priorizó la estandarización y estableció controles de calidad. Frente a la gran popularización de versiones no "auténticas" de la comida tailandesa que, según las autoridades, podría dañar su valoración por parte de los consumidores.

Para ello, creó el certificado oficial "Thai Select" para ser otorgado a aquellos restaurantes de comida tailandesa en el extranjero que reuniesen los estándares de calidad de comida y servicio pretendidos y que permitieran de este modo apreciar la autenticidad de la experiencia gastronómica original tailandesa (Benjasirichai, 2005).

El certificado, que tiene una validez de tres años a ser renovado periódicamente, es otorgado por el Departamento de Promoción del Comercio Internacional del Ministerio de Comercio, luego de que la Autoridad de Turismo de Tailandia presenta los establecimientos seleccionados. Estos últimos deben reunir un conjunto de requisitos, entre los que se encuentran haber estado operando, por lo menos, con seis meses de antigüedad y estar abierto, minimamente, cinco días a la semana.

Se exige también que empleen a cheftailandeses experimentados o cocineros con más de dos años de experiencia, al menos poseer certificado de 
una institución acreditada en la preparación de comida tailandesa. Entre los requisitos también se encuentra la exigencia de tener al menos seis platos tailandeses en el menú, que utilicen recetas aprobadas, ingredientes autóctonos tailandeses sin adaptación, y, finalmente, que acepten recibir la inspección de los agentes del Departamento de Promoción del Comercio Internacional, o, en caso contrario, de la embajada de Tailandia en el país. En el año 2004 se habían otorgado 698 certificados "Thai Select" a restaurantes de comida tailandesa en el extranjero. Para el 2015 ya se habían otorgado 1.135 (Juyan Zhang, 2015, p.11; Ichijo y Ranta, 2015, p.112).

Esta iniciativa diplomática buscó potenciar la complementación entre la gastronomía, el turismo, la producción de alimentos y el comercio internacional. Se buscó generar una sinergia positiva que redundaría en más beneficio económico para estos sectores productivos y mayores ingresos de divisas para el país.

Como se planteó anteriormente, este programa tuvo como finalidad principal incrementar la cantidad de turistas internacionales que visitasen el país, a partir de la cual apuntalar todavía más su posición como uno de los principales destinos turísticos del mundo.

Estos nuevos visitantes significarían un nuevo incentivo a la industria gastronómica del país, además de otros incentivos económicos positivos para el conjunto de la industria de turismo. Entendiendo que una campaña turística convencional promociona al país por medio de imágenes de la belleza de los paisajes naturales y el encanto de los sitios históricos, una campaña gastronómica cumple con esos objetivos a través de los sabores, los aromas y las texturas de la comida. Acompañando este programa tuvieron lugar el lanzamiento de nuevas campañas turísticas por parte del gobierno tailandés ${ }^{11}$.

Estas campañas buscaron fomentar el sector turístico para recuperarse de los efectos económicos negativos experimentados por un conjunto de crisis socio-políticas y económicas, como también desastres naturales. Desde la crisis económica de 1997-1998, la epidemia en la región del Síndrome Respiratoria Agudo Grave en 2002-2003, la gripe aviar en 2004-2006 hasta el impacto global del tsunami del Océano Índico del 2004, sin mencionar

11. En el 2003 lanzó la campaña turística bajo el slogan "Unseen Thailand" ("Tailandia Oculta"), buscando sugerir las atracciones todavía desconocidas que Tailandia ofrece a quienes la visiten, que vendría a continuación de la campaña turística de 1998 bajo el slogan "Amazing Thailand" ("Tailandia Sorprendente"), que buscaba sugerir las atracciones extraordinarias que el país tiene a quienes lo visitan. Esta campaña sería sucedida por las campañas turísticas "Thailand Grand Invitation" ("Tailandia Gran invitación") en el 2006, "Thailand Talk to the World" ("Tailandia Habla al Mundo") en el 2007, "Amazing Thailand Always Amazes You" ("La Sorprendente Tailandia Siempre te Sorprende") en el 2010, y "Amazing Thailand it Begins with the People" ("La Sorprendente Tailandia Comienza con las Personas") en el 2013. (Tourism Authority of Thailand 2015, Ibid). 
una serie de ataques terroristas, que redujeron drásticamente los viajes internacionales. Este programa buscó repercutir además en un incremento de las exportaciones de productos alimenticios de Tailandia, facilitando la apertura y el acceso a nuevos mercados.

En el 2003 el gobierno tailandés lanzó la campaña "Thailand. The Kitchen of the World" ("Tailandia: La Cocina del Mundo") a partir de la cual buscaba no sólo mostrar la riqueza gastronómica, también apuntaba a promocionar la industria de alimentos del país para apuntalar su posición como uno de los principales exportadores de alimentos del mundo.

El Ministerio de Agricultura reformó los controles fitosanitarios para asegurar la calidad de los alimentos exportados del país. Los restaurantes de comida tailandesa en el extranjero incentivaron las exportaciones de ingredientes y condimentos autóctonos, especialmente especias, hierbas y vegetables, como también otros artículos decorativos típicos y de otros productos específicos de Tailandia (Sunanta, 2005).

Los gobiernos sucesores de Thaksin Shinawatra adoptaron nuevas políticas económicas que desincentivaron algunas de las iniciativas anteriormente mencionadas, incluidas las turísticas (Cohen, 2008). Estas políticas serían retomadas y reforzadas nuevamente durante el gobierno de la Primera Ministro Yingluck Shinawatra (2011-2014), hermana de Thaksin, en el marco de la campaña "Thailand Kitchen to the World" "Tailandia, Cocina al Mundo") lanzada en el 2012 en Australia. Recientemente se estableció el Comité de la Delicia Tailandesa por parte del nuevo gobierno tailandés ${ }^{12}$.

\section{Conclusiones}

Las páginas anteriores mostraron la activa presencia del Estado para fomentar la promoción de la comida tailandesa en el mundo como una expresión de la cultura nacional. A su vez, a través de esta, se promocionaba turísticamente el país en todo el mundo. La diplomacia asiste al turismo gastronómico, al promocionar al país como destino gastronómico.

El gobierno de Tailandia fue pionero en aprovechar el recurso no convencional de la popularidad de la comida tailandesa en el mundo e instrumentalizarlo estratégicamente dentro de las iniciativas diplomáticas llevadas adelante para relacionarse internacionalmente con otros estados y los pueblos que representan.

De este modo no sólo tuvo la visión, sino también la inteligencia de aprovecharlo de una manera holística, entendiendo la complejidad de

12. "Thai Tasting Robot tastes for Authenticity", Bangkok, BBC News. Extraído de http://www.bbc.com/news/ en diciembre de 2015. 
interacciones sinérgicas que puede tener con otros aspectos de la vida nacional, desde beneficios económicos por la promoción del turismo y el incentivo de las exportaciones, hasta efectos políticos por la construcción de la identidad nacional y el estímulo del sentimiento nacional de orgullo internacional del pueblo tailandés.

De aquí que el esfuerzo no fue sólo de una agencia gubernamental o ministerio, sino que se trata de una política de Estado involucrando a diversos organismos del Estado tailandés, como el Ministerio de Relaciones Exteriores, Ministerio de Comercio, Ministerio de Agricultura, Ministerio de Trabajo, entre otros. De aquí la denominación del programa como "Global Thai".

Aunque aprovechada y apuntalada por el gobierno tailandés, la popularidad de la cocina tailandesa en el mundo se debe a un proceso de internacionalización mucho más complejo, donde incidieron desde grandes procesos históricos hasta intereses concretos que escapan al control inmediato del Estado tailandés.

Es así que la presencia de las tropas estadounidenses en territorio tailandés en su iniciativa militar contra las fuerzas comunistas en el territorio vietnamita en el contexto de la Guerra Fría fue la chispa que explotó la masificación de la industria del turismo internacional en Tailandia. También los flujos de migración tailandesa a países occidentales en busca de mejores oportunidades, lo que permitió el establecimiento de los primeros restaurantes de comida tailandesa fuera del país, destinados primero a la comunidad tailandesa y luego a los consumidores occidentales no tailandeses.

De esto se desprende la multiplicidad de actores, desde individuos emprendedores privados hasta agencias gubernamentales públicas, que participaron y participan en el sostenimiento de la popularidad de la comida tailandesa en el mundo. Y tambien la gran complejidad de interacciones entre distintos procesos, que llevaron a la comida tailandesa a ser una de las comidas étnicas más reconocidas internacionalmente.

\section{Bibliografía}

Anderson, B. (1993), "Comunidades Imaginadas. Reflexiones sobre el Origen y la Difusión del Nacionalismo”, México, Fondo de Cultura Económica.

Benjasirichai, D. (2005). "Laws and Regulations Influencing Thai Restaurants: A Case Study in the Netherlands", en Petchsiri, A., Roth, W., y Raveepaopong, R. (eds.), Asia Impressions on the European Union in 2003-2004, Bangkok, Chulalongkorn University Printing House. 
Beasley, T., Hirsch, P., Rungmanee, S. (2014). Thailand in Australia. Sydney, University Publishing Managed Service of the University of Sydney.

Bianco, L. (1995). “Asia Contemporánea”, México, Siglo XXI Editores.

Chapple-Sokol, S. (2013). "Culinary Diplomacy: Breaking Bread to Win Hearts and Minds” The Hague Journal of Diplomacy, Hague (pp. 161-183).

Cohen, E. (2008). Explorations in Thai Tourism. Collected Case Studies. Bingley, Emerald Group Publishing.

Douglas, N., y Douglas N. (2011). "Tourism in South and Southeast Asia: Historical Dimensions", en Hall, C. M. y Page, S. (eds.), Tourism in South and Southeast Asia: Issues and Cases, Nueva York, Routledge.

Ferguson, P. P., \& University Press Scholarship Online - Frontfile. (2004). Accounting for taste: The triumph of french cuisine. Chicago: The University of Chicago Press

Higham, J. (2011). “Thailand: Prospects for a Tourism-led Economic Recovery”, en Hall, C. M. y Page, S. (eds.), "Tourism in South and Southeast Asia: Issues and Cases" (pp. 129-143), Nueva York, Routledge.

Hoeffel, E. M., Rastogi, S., Kim, M. O., y Shahid, H. (2012). The Asian Population: 2010 Census Briefs. Washington, U.S. Census Bureau, Economics and Statistics Administration, U.S. Department of Commerce.

IchiJo, A., y Ranta, R. (2015). Food, National Identity and Nationalism: From Everyday to Global Politics. Londres, Palgrave Macmillan.

InTARAKUMNERD, P. (2011). Thaksin's Legacy: Thaksinomics and its Impacts on Thailand's National Innovation System and Industrial Upgrading (pp. 31-6o), Kuala Lumpur, International Journal of Institutions and Economics, $3(1)$.

Kontogeorgopoulos, N. (1998), Tourism in Thailand: Patterns, Trends, and Limitations (pp. 225-238), Nueva York, Pacific Tourism Review, 2.

Nirwandy, N., y Awang, A. A. (2013), Conceptualizing Public Diplomacy Social Convention Culinary: Engaging Gastro Diplomacy Warfare for Economic Branding (pp. 325-332), Procedia - Social and Behavioral Sciences.

PanikKar, K. M. (1966). Asia y la Dominación Occidental. Un Examen de la Historia de Asia desde la llegada de Vasco da Gama (1498-1945)”, Buenos Aires, Editorial Universitaria de Buenos Aires.

Rockower, P. S. (2012), “Recipes for Gastrodiplomacy” (pp. 235-46), Place Branding and Public Diplomacy, 8(3).

Si Qian NG, C. (2015). "Culinary Diplomacy and Nationalism. Japan and Thailand" January 17, 2015. 
Asia

América

Latina

66

Thongrhon Promsaka Na Sakolnakorn (2012), "A Study Of The Management Of Thai Food Shops In Auckland, New Zealand” (pp. 193-196), Littleton, The Clute Institute, Journal of Business Case Studies, 8(2).

Sunanta, S. (2005), “The Globalization of Thai Cuisine”, Toronto, Canadian Council for Southeast Asian Studies Conference.

VAN Esterik, P. (2008), "Food Culture in Southeast Asia”, Westport, Greenwood Press.

(2011), "Thailand", en Abdala, K. (ed.), "Food Cultures of the World Encyclopedia. Asia and Oceania” (pp. 253-261), Santa Bárbara, ABC-CLIO.

VilLIERS, J. (1973), “Asia Sudoriental. Antes de la Época Colonial”, Madrid, Siglo XXI de España Ed.

JuYan Zhang (2015), "The Foods of the Worlds: Mapping and Comparing Contemporary Gastrodiplomacy Campaigns" (558-591), Los Angeles, University of Southern California USC Annenberg Press, International Journal of Communication, 9. 

